\title{
PADRÕES DE CRESCIMENTO DE Araucaria angustifolia E Cedrela fissilis NO CENTRO-SUL DO PARANÁ
}

\section{LIFETIME GROWTH PATTERNS OF Araucaria angustifolia AND Cedrela fissilis IN SOUTH CENTER REGION OF PARANÁ}

\author{
Amanda Köche Marcon ${ }^{1}$, Tomaz Longhi-Santos², Paulo Cesar Botosso3, \\ Christopher Thomas Blum ${ }^{4}$, Kelly Geronazzo Martins ${ }^{5}$, Franklin Galvão ${ }^{6}$ \\ 1, 2, 4,6 Universidade Federal do Paraná, Curitiba, Paraná, Brasil-amandakoche@gmail.com, \\ tomazlonghi@gmail.com, ctblum.ufpr@gmail.com\&fgalvao@ufpr.br \\ ${ }^{3}$ EMBRAPA Florestas, Colombo, Paraná, Brasil - paulo.botosso@embrapa.br \\ ${ }^{5}$ Universidade Estadual do Centro-Oeste, Irati, Paraná, Brasil - kellygm77@gmail.com
}

RESUMO

\begin{abstract}
A avaliação dos padrões de crescimento das árvores pode resgatar informações e indicar características do comportamento ecológico das espécies, importantes tanto para a conservação quanto para o seu manejo. A hipótese deste estudo é que os ciclos de corte de florestas do Sul do país resultaram em eventos de liberação do crescimento nas árvores remanescentes. Os objetivos foram identificar os padrões de crescimento, compará-los com a autoecologia das espécies e verificar o sincronismo dos eventos de liberação ou supressão. Foram coletadas 3 amostras não destrutivas de lenho de cada uma das 30 árvores por espécie, com uso de trado de incremento. As amostras de lenho foram secas e polidas, para posterior delimitação e mensuração da largura dos anéis anuais de crescimento. Para verificar alterações no incremento, foram calculados os incrementos diamétricos relativos, buscando detectar padrões de liberação e/ou supressão do crescimento, bem como a sincronicidade destes eventos. Os resultados indicaram diferentes padrões de crescimento para ambas as espécies. Para A. angustifolia, a maioria das árvores não registrou eventos de liberação ou supressão. Para $C$. fissilis, a maioria das árvores passou por, pelo menos, um evento de supressão. A sincronicidade entre eventos de liberação e supressão foi baixa, indicando que eventos individuais, e não de grande escala, atuaram. Não foi possível identificar liberações do crescimento relacionadas ao ciclo histórico de corte seletivo ocorrido na região Sul do Brasil.
\end{abstract}

PALAVRAS-CHAVE: Anéis de crescimento, Crescimento relativo, Dendrocronologia, Floresta Ombrófila Mista, Liberação e supressão.

\section{ABSTRACT}

Lifetime growth patterns analysis could retrieve past data and indicate species ecological behavior, essential information for nature conservation and sustainable use. We hypothesized that trees have a growth pattern according to their light demand and the cutting cycles of natural forests in the southern region resulted in growth releases. Our main objectives were to identify the lifetime growth patterns, compare with species autecology and check synchronously of suppressions and releases. We collected three wood samples from 30 trees per species using an increment borer. Wood samples were dried and polished for subsequent measurement of tree ring width. To verify changes in medium-term, we calculated the percentage growth change for year, seeking to detect suppressions, releases and their synchronicity. Our results indicated different growth patterns. For $A$. angustifolia, most of trees did not experience releases or suppressions. For $C$. fissilis, most of trees experienced at least one suppression. Releases and suppressions synchronously were low, indicating that individual and non-large-scale events have acted on radial growth. It was not possible to identify growth releases related with historic cutting cycles in the southern region of Brazil.

KEYWORDS: Dendrochronology, Ombrophilous Mixed Forest, Relative tree growth, Suppression and release, Tree rings. 


\section{INTRODUÇÃO}

Avaliar a trajetória de crescimento das árvores pode resgatar valiosas informações de eventos do passado, seja com objetivo de estudar a autoecologia das espécies, ou, para determinar a necessidade da aplicação de tratamentos silviculturais (DE RIDDER et al., 2013; ROZENDAAL \& ZUIDEMA, 2011). A obtenção destas informações pode ser feita utilizando técnicas de dendrocronologia, considerando que os anéis de crescimento atuam como uma ferramenta na detecção de mudanças ambientais (COOK \& KAIRIUKSTIS, 2013). O fato de os eventos ambientais serem registrados nos anéis anuais de crescimento é de grande importância para as ciências históricas, uma vez que permite datar eventos dentro de um ano calendário e recuperar informações não registradas previamente (SCHWEINGRUBER, 2012). A partir dos anéis de crescimento é possível entender como o crescimento das árvores é afetado, tanto retrospectiva quanto prospectivamente (BOWMAN et al., 2013) e detectar períodos de maior ou menor crescimento (FRITTS, 1976; SCHWEINGRUBER, 2012).

Identificar eventos que causam um grande crescimento (liberações) ou uma redução no crescimento (supressões), é essencial para entender não só as diferenças na história de vida das espécies, como entre indivíduos de uma mesma espécie (BRIENEN \& ZUIDEMA, 2006). A supressão do crescimento é comum em árvores jovens vivendo no sub-bosque da floresta, onde há menos luz disponível e maior competição (BRIENEN et al., 2006). Já árvores que estão no dossel podem sofrer supressões, por exemplo, em função alterações climáticas - que podem envolver de longos períodos de déficits hídricos - ou em como resultado de uma infestação por parasitas (BRIENEN et al., 2006). Eventos de liberação acontecem, geralmente, quando há uma abertura no dossel da floresta, permitindo a entrada de luz, ou quando há uma expressiva redução na competição por alguma outra razão (BRIENEN \& ZUIDEMA, 2006; BRIENEN et al., 2006; GUREVITCH et al., 2009). A redução na competição pode ocorrer em casos de corte seletivo da vegetação, como ocorreu nas décadas de 1980 e 1990 em muitos fragmentos da Floresta Ombrófila Mista (SEVEGNANI et al., 2013).

Estudos relacionados ao padrão de crescimento (liberações e supressões) ainda não são comuns no Brasil. Atualmente, foram desenvolvidos alguns estudos na Amazônia, voltados principalmente para o manejo florestal (e.g., BRIENEN et al., 2010; GROGAN \& LANDIS, 2009; SCHÖNGART et al., 2015) e na Mata Atlântica, no Sul do país (SPATELF et al., 2009), buscando identificar perturbações na história individual das árvores. Sendo assim, têm-se poucas informações de como são os padrões de crescimento das árvores de florestas tropicais e subtropicais do Brasil em longo prazo (BAKER \& BUNYAVEJCHEWIN, 2006).

Dentro deste contexto, este estudo possui os objetivos de: i) identificar os padrões de crescimento das espécies; ii) comparar com seu padrão autoecológico; e iii) verificar se existe sincronismo nos eventos de liberação ou supressão do crescimento entre os indivíduos. A hipótese que norteia este estudo é que os ciclos históricos de corte de florestas nativas na região resultaram em liberação do crescimento das árvores suprimidas.

\section{MATERIAL E MÉTODOS}

\section{Área de estudo}

O estudo foi realizado no Terceiro Planalto Paranaense, município de Pinhão, área cedida à Companhia Paranaense

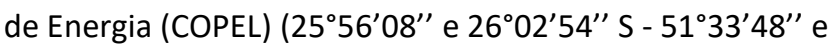
$51^{\circ} 38^{\prime} 47^{\prime \prime}$ W). O regime climático na região é do tipo $\mathrm{Cfb}$, apresentando chuvas bem distribuídas, verões brandos e inverno com ocorrência de geadas (ALVARES et al., 2013). A precipitação média anual é de $1.745 \mathrm{~mm}$, a temperatura média do mês mais frio fica abaixo de $14^{\circ} \mathrm{C}$ e a temperatura média no mês mais quente abaixo de $23^{\circ} \mathrm{C}$ (KNMI, 2017; SNIRH, 2017). A fitofisionomia é caracterizada como Floresta Ombrófila Mista Montana, possuindo composição florística de gêneros primitivos como Drymis, Araucaria e Podocarpus (IBGE, 2012).

O relevo na região é bastante movimentado, com altitudes variando de 700 a 1.250 m.s.n.m. (MIRANDA, 2009). A formação geológica dominante é a Serra Geral, constituída principalmente de basaltos intercalados de material sedimentar, como arenitos e siltitos (MINEROPAR, 2006). Os solos apresentam como características cores amareladas ou brunadas, com grande quantidade de matéria orgânica e fertilidade baixa, predominando os Latossolos, Nitossolos e Cambissolos (BEHRING et al., 2008)

\section{Espécies estudadas}

Foi avaliado o crescimento diamétrico de duas espécies com ocorrência natural na área de estudo, Araucaria angustifolia (Bertol.) Kuntze (pinheiro-brasileiro) e Cedrela fissilis Vell. (cedro-rosa). A seleção destas espécies se deu em função de características como longevidade (KLEIN, 1960; REITZ; KLEIN \& REIS, 1978) e reconhecido potencial 
para estudos com anéis de crescimento (BAKER et al., 2017; WEHR \& TOMAZELLO-FILHO, 2000), possibilitando comparar as repostas das duas espécies ao ambiente.

\section{Coleta dos dados}

De cada árvore, 2 a 3 raios diametralmente opostos foram extraídos com uso de trado de incremento $(5 \mathrm{~cm}$ de diâmetro), a $1,30 \mathrm{~m}$ de altura do solo (DAP). Foram selecionados para cada espécie 30 indivíduos adultos com DAP $\geq 40 \mathrm{~cm}$, em condições semelhantes de sítio.

As amostras radiais de lenho foram secas em temperatura ambiente, fixadas sobre suportes de madeira e polidas com uso de lixas abrasivas de diferentes granulometrias (80 a 1.000 grãos $/ \mathrm{cm}^{2}$ ), tendo sido descartadas as amostras consideradas inviáveis por não terem o lenho íntegro (e.g. deformidades e/ou quebras durante a secagem). Os anéis de crescimento foram reconhecidos com auxílio de uma lupa estereomicroscópica, sendo cada um destes delimitado e sincronizados visualmente. As amostras de lenho foram digitalizadas (resolução de 1.200 dpi) e a medição da largura dos anéis de crescimento foi feita com o uso do software de processamento de Image Pro Plus versão Free Trial (MEDIA CYBERNETICS, 2017).

\section{Crescimento diamétrico relativo}

Com a finalidade de verificar eventuais alterações no incremento no médio prazo, foram calculados os crescimentos diamétricos relativos, buscando detectar padrões de liberação e de supressão de crescimento. 0 crescimento relativo foi calculado conforme Nowacki \& Abrams (1997), utilizando um período de 10 anos:

$$
C R(\%)=\frac{(M 2-M 1)}{M 1} * 100
$$

Em que: CR (\%) corresponde ao crescimento relativo no período; $\mathrm{M} 1$ corresponde à média do incremento diamétrico no período i; e, M2 corresponde ao incremento médio no período $\mathrm{i}+1$.

O crescimento diamétrico relativo foi calculado tomando por base as séries de crescimento diamétrico de cada árvore, excluindo-se as primeiras e as últimas décadas, que não podem ser calculadas por definição do índice (BAKER \& BUNYAVEJCHEWIN, 2006).

Eventos de liberação e de supressão de crescimento diamétrico foram considerados de acordo com o sugerido por Brienen \& Zuidema (2006). Segundo estes autores, a liberação de crescimento ocorre quando o crescimento relativo supera em mais de $100 \%$ a taxa média de crescimento durante pelo menos cinco anos. Por outro lado, o evento de supressão de crescimento ocorre quando há uma redução relativa de $50 \%$ na taxa média de crescimento do diâmetro durante pelo menos cinco anos subsequentes.

Os padrões de trajetória de crescimento em diâmetro para cada árvore foram classificados, conforme sugerido por Brienen \& Zuidema (2006) em: (1) Nenhuma liberação ou supressão: árvore chega ao dossel sem a ocorrência de grandes mudanças no crescimento. É aceita apenas uma liberação de crescimento (< 5 anos); (2) Uma liberação: ocorre um forte evento de liberação de crescimento (> 5 anos) durante o acesso do indivíduo ao dossel, sendo que as árvores mostram um evento de lançamento, que pode ser precedido por supressão; (3) uma supressão: ocorrência de um evento de supressão, sendo que as árvores geralmente têm uma taxa de crescimento inicial alta, seguida por uma forte diminuição do crescimento e nenhuma liberação de crescimento subsequente; e (4) múltiplas liberações e supressões: o crescimento ocorre através de múltiplas liberações e supressões, sendo que as liberações devem ser sempre seguidas por uma supressão do crescimento e/ou separadas de uma nova liberação de crescimento em mais de 5 anos. Para melhor visualização dos períodos de supressão e liberação do crescimento, as árvores foram agrupadas de acordo com o tamanho das séries.

A sincronicidade das supressões e liberações foi avaliada calculando a proporção de árvores com eventos que começaram no mesmo ano, com flexibilidade de mais ou menos um ano $( \pm 1)$.

\section{RESULTADOS E DISCUSSÃO}

A análise do crescimento foi realizada com 25 árvores de $A$. angustifolia e 26 de $C$. fissilis, de um total inicial de 30 indivíduos por espécie. Amostras de lenho de 5 árvores de $A$. angustifolia e de quatro árvores de $C$. fissilis foram descartadas em função de deformidades e/ou danos que impediram a delimitação e mensuração da largura dos anéis de crescimento.

Comparando os valores de incremento médio por ano com outros estudos realizados em regiões próximas, observou-se que, para C. fissilis, foram encontrados maiores incrementos médios anuais (Tabela 1). Em Irati, Stepka (2012) encontrou um incremento médio de 4,0 $\mathrm{mm} / \mathrm{ano}$; em Teixeira Soares e Fernandes Pinheiro, Figueiredo Filho et al. (2010) observou 3,6 mm/ano e em 
São João do Triunfo, Schaaf et al. (2005) observou 4,8 $\mathrm{mm} / \mathrm{ano}$. As caracteristicas do sítio, como a disponibilidade de nutrientes e as condições edáficas (litologias diversas), bem como as particularidades dos indivíduos, como a sua posição sociológica e os níveis de competição, podem explicar essas diferenças (ASSMANN, 1970; GUREVITCH et al., 2009; KOZLOWSKI \& PALLARDY, 1997).

Tabela 1. Incremento médio anual em diâmetro (IMA), desvio padrão (DP) e idade dos indivíduos amostrados.

\begin{tabular}{cccccc}
\hline \multirow{2}{*}{ Espécie } & \multicolumn{2}{c}{ IMA } & DP & \multicolumn{3}{c}{ Idade (anos) } \\
\cline { 2 - 6 } & \multicolumn{2}{c}{ (mm/ano) } & média & máxima & mínima \\
\hline A. angustifolia & 8,36 & 3,94 & 94 & 262 & 41 \\
C. fissilis & 6,43 & 2,46 & 74 & 127 & 43 \\
\hline
\end{tabular}

Para A. angustifolia, valores de incremento médio obtidos em Santa Catarina, em Lages e Painel, 7,03 e 9,08 mm/ano, respectivamente (MINATTI, 2015; RICKEN, 2014), são próximos aos valores encontrados neste estudo, indicando que as taxas de crescimento encontradas estão dentro do esperado.

Os resultados da análise do crescimento relativo dos indivíduos de $A$. angustifolia indicaram diferentes padrões de crescimento. Dez indivíduos (40\%) tiveram trajetória do tipo 1, sem nenhum evento de liberação ou supressão; dois indivíduos (8\%) tiveram trajetória do tipo 2, com uma liberação; oito indivíduos (32\%) passaram por uma supressão sem liberação, tipo 3; e cinco indivíduos (20\%) tiveram múltiplas liberações e supressões durante o crescimento, sendo classificados no tipo 4 (Figura 1).

A maioria das árvores de $A$. angustifolia não passou por nenhum evento de liberação ou supressão ao longo da vida. Possivelmente, estas árvores cresceram sem nenhum impedimento para alcançar o dossel, desenvolvendo-se em condições de alta luminosidade (BRIENEN \& ZUIDEMA, 2006). Estas situações podem ocorrer nos casos em que a árvore cresceu em uma grande clareira ou em ambientes abertos (CLAESSENS et al., 2006), enquanto a ocorrência de um período de supressão sugeriria que a árvore estava crescendo no sub-dossel (CHERUBINI et al., 1998).

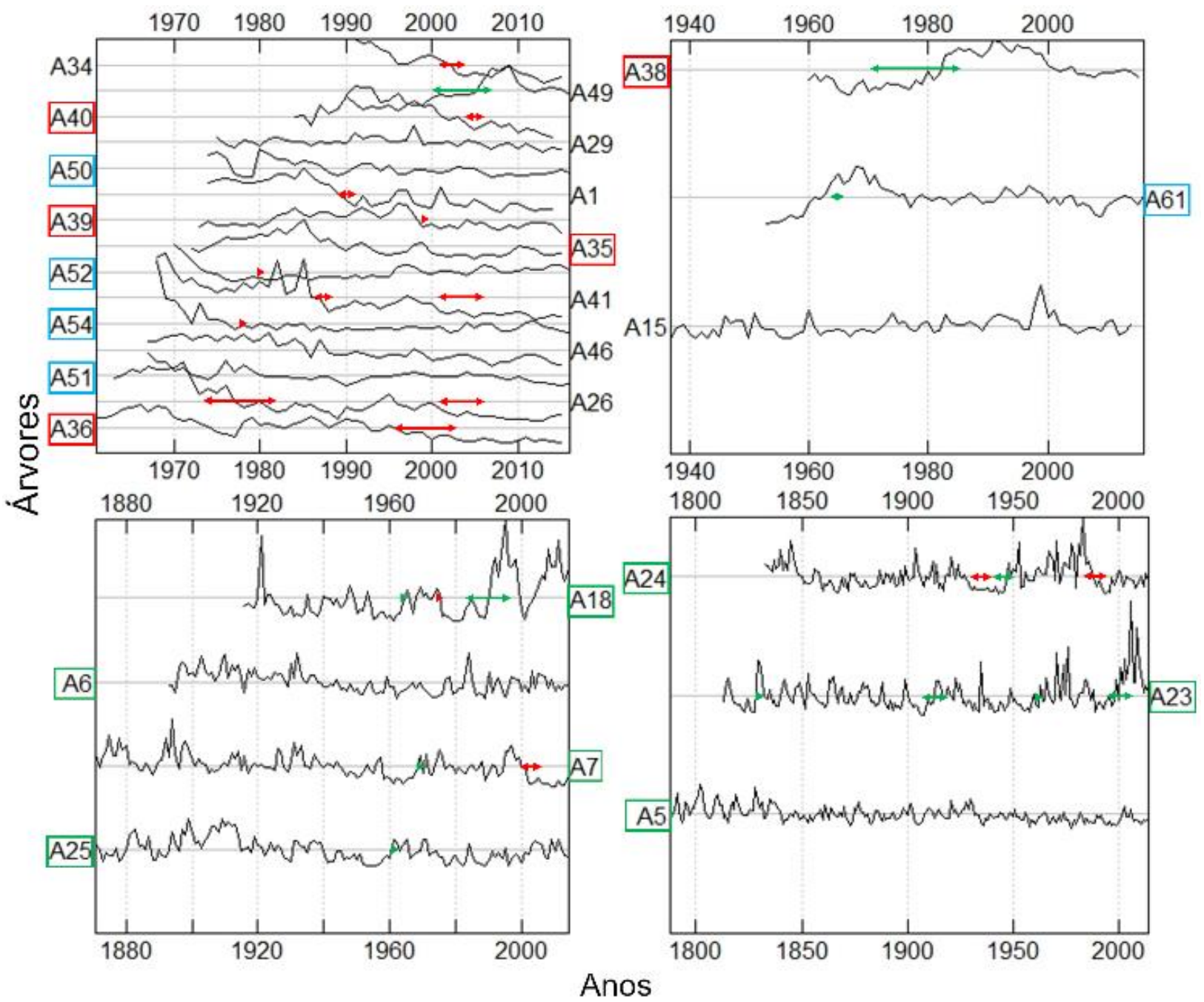

Figura 1. Séries temporais individuais de A. angustifolia apresentando os eventos de supressão (setas vermelhas) e liberação do crescimento (setas verdes). Árvores localizadas próximas umas das outras foram identificadas com a mesma cor. 
Provavelmente 0 padrão encontrado para $A$. angustifolia está ligado à época de exploração madeireira na região. Nesse período, outras espécies de valor comercial foram suprimidas, muitas vezes restando grandes capões compostos quase que somente por $A$. angustifólia, possibilitando que estas árvores cresçam livremente. Dentro da floresta, a espécie possui um padrão de ocorrência em coortes, onde os indivíduos que nasceram em anos próximos ficam no sub-bosque aguardando uma abertura de luz para alcançar o dossel, padrão também chamado de distribuição multimodal (EBLING \& NETTO, 2015; GOMES, 2015). Esse tipo de distribuição é formado em decorrência de agentes antrópicos e naturais que atuam nos nichos (EBLING \& NETTO, 2015). Espera-se, nesta situação, que as espécies passem por pelo menos um evento de liberação do crescimento, quando ocorrer alguma mudança na floresta e as árvores atinjam o dossel (BRIENEN \& ZUIDEMA, 2006; SILVA et al., 2009).

Ainda que a maioria das árvores de $A$. angustifolia não tenha passado por eventos de liberação ou supressão, as diferentes estratégias de vida da espécie resultaram em diferentes padrões de crescimento, com alguns indivíduos experenciando liberações de crescimento. Os resultados reforçam a capacidade de adaptação da espécie, podendo sobreviver tanto em clareiras ou a pleno sol, quanto em ambientes sombreados, onde permanece, até que uma abertura de clareira ocorra e possa alcançar o dossel (FRANCO \& DILLENBURG, 2007; INOUE \& TORRES, 1980). A diversidade de estratégias de vida demonstra a plasticidade da espécie, particularmente pela otimização da captura de luz (VALLADARES \& NIINEMETS, 2008).
Já os indivíduos de $C$. fissilis, demonstraram em sete árvores (26,9\% dos casos), trajetória do tipo 1 (não passaram por nenhum evento de liberação e supressão); em outras sete árvores $(26,9 \%)$ a trajetória foi do tipo 2 , com uma liberação; nove árvores apresentaram trajetória tipo $3(34,6 \%)$, com uma supressão sem liberação e três árvores foram do tipo 4, com múltiplas liberações e supressões (11,5\%) (Figura 2).

As trajetórias de crescimento de C. fissilis foram diversas. Contudo, muitas árvores tiveram, ao longo da vida, um evento de liberação do crescimento seguido de supressão ou não. Os resultados seguem um padrão de comportamento ligado a autoecologia da espécie, que podem possuir um comportamento umbrófilo na fase juvenil, conforme citado por Amaral (2006) e Martins \& Lago (2008). Pode-se inferir que estas árvores tiveram eventos de liberação ao passarem de uma condição sombreada, no seu estabelecimento, para uma condição de alta luminosidade, durante o acesso ao dossel (BAKER \& BUNYAVEJCHEWIN, 2006; SCHÖNGART et al., 2015).

Segundo Gandara (2009), as plantas desta espécie possuem capacidade de permanecer em sub-bosque, com crescimento lento, aguardando uma abertura no dossel. As exigências fisiológicas da espécie contemplam certa quantidade de sombra para seu desenvolvimento (INOUE, 1977). A ocorrência de eventos de supressão do crescimento pode estar relacionada a ocorrência de ataques da broca-do-cedro (Hypsipyla grandella), especialmente no início da vida de indivíduos em áreas de clareira e de menor densidade de árvores (PEREIRA et al., 2016).

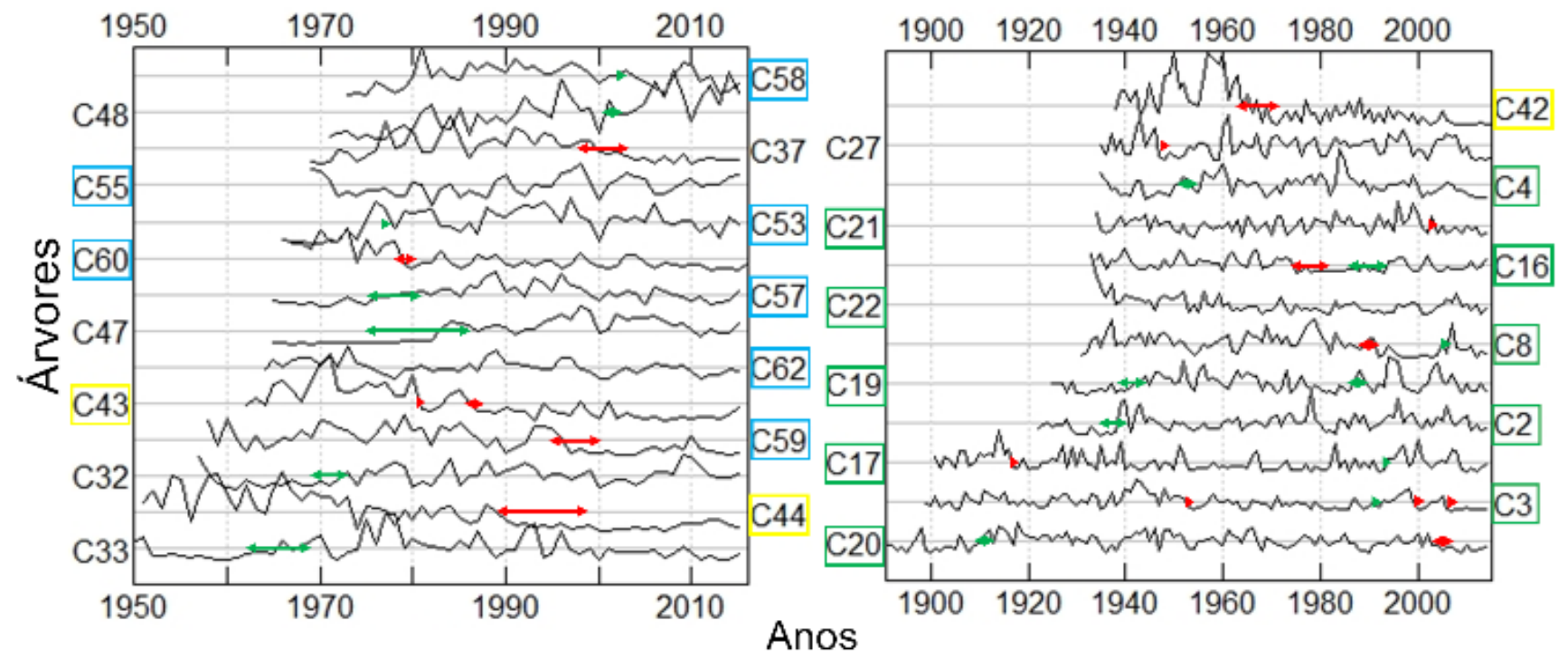

Figura 2. Séries temporais individuais de $C$. fissilis apresentando os eventos de supressão (setas vermelhas) e liberação (setas verdes). Árvores localizadas próximas umas das outras foram identificadas com a mesma cor. 
O teste de sincronicidade demonstrou que para as duas espécies simultaneidade de eventos de liberação e supressão foi baixa (Figuras 3 e 4). Para A. angustifolia, o período com maior sincronia na liberação de crescimento ocorreu entre 1962-1964 (33,3\%), porém apenas nove árvores fazem parte deste conjunto de dados. Para os eventos de supressão do crescimento, a maior sincronia entre árvores ocorreu no ano 2000, com 24\% de 25 indivíduos apresentando supressão.

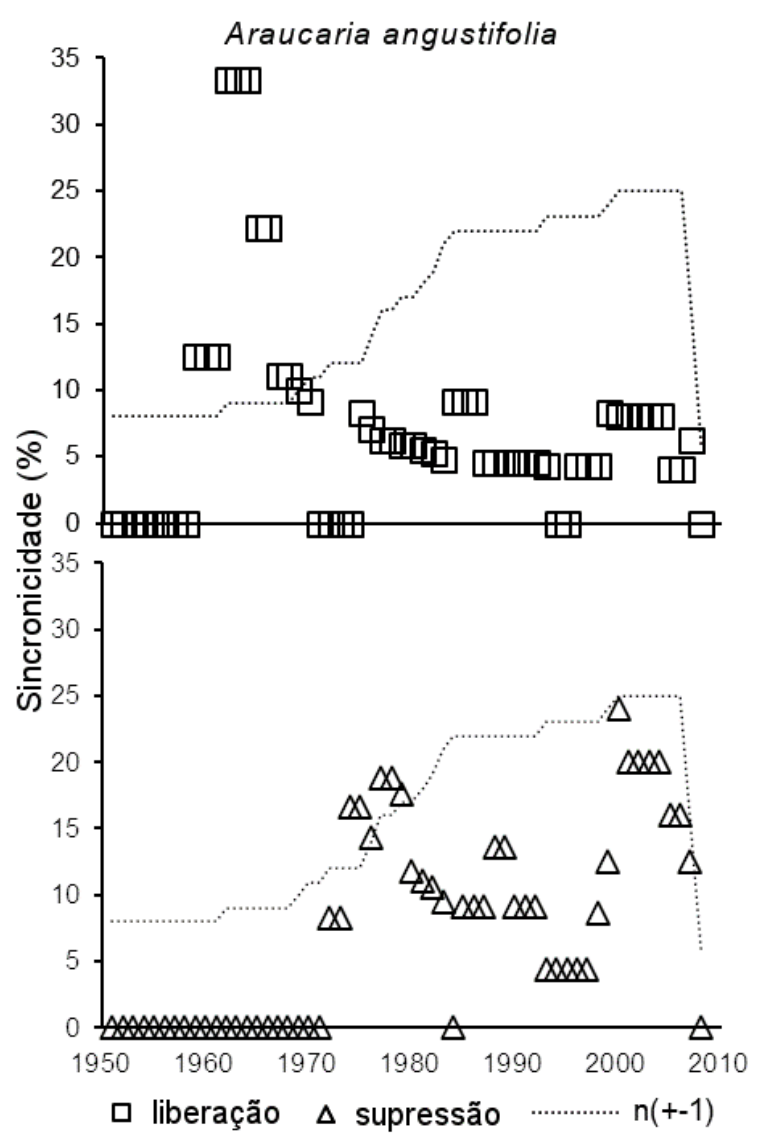

Figura 3. Sincronicidade dos eventos de liberação e supressão para $A$. angustifolia.

Entre as árvores de $C$. fissilis, as liberações tiveram uma sincronicidade máxima entre os anos de 1982-1984, onde $15,4 \%$ dos indivíduos passaram por um evento de liberação. Os eventos de supressão ocorreram em, no máximo, 19,2\% dos indivíduos, entre os anos 2005-2006.

A baixa sincronicidade entre eventos de liberação e supressão não revelaram claras evidências de mudanças drásticas nos padrões do crescimento induzidas por eventos extremos no clima (BRIENEN et al., 2010). Mesmo árvores próximas não tiveram alto sincronismo de eventos de liberação ou supressão do crescimento, não confirmando a teoria de que os padrões de crescimento das árvores são sincronizados em áreas relativamente grandes (SZWAGRZYK \& SZEWCZYK, 2001).

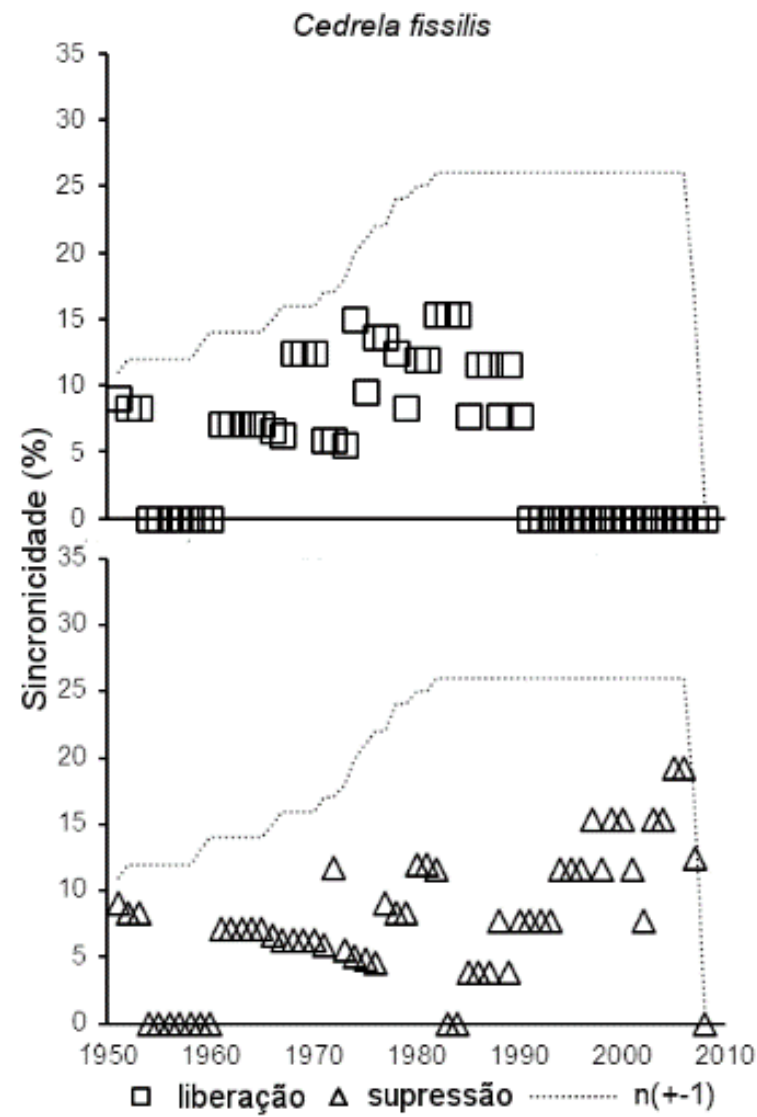

Figura 4. Sincronicidade dos eventos de liberação e supressão para $C$. fissilis.

Também não foi identificado um alto sincronismo de liberação do crescimento concomitantes ao período de exploração florestal na região, entre as décadas de 1980 e 1990; onde houve corte seletivo ou mesmo total em muitos fragmentos da Floresta Ombrófila Mista (SEVEGNANI et al., 2013). Se esperaria este resultado em função da redução da competição após corte seletivo (WEHR \& TOMAZELLO-FILHO, 2000). Os resultados indicam que eventos individuais, e não de grande escala, atuaram em supressões ou liberações. A abertura de uma pequena clareira que possibilite maior crescimento de algumas árvores no local, a ocorrência de injúrias ou ataques por patógenos ou pragas e a ocorrência de raios e/ou quebras na copa por outras razões, são exemplos de eventos que podem ser individuais e afetar apenas as árvores do micro sítio, levando a trajetórias de crescimento divergentes (LLORET et I., 2011; SVOBODA et al., 2011; DI FILIPPO et al., 2017).

Por outro lado, observa-se um aumento do número de supressões para as duas espécies - próximo aos anos 19952000, mas de forma mais expressiva para C. fissilis. A maioria das árvores passou por pelo menos uma supressão, indicando a habilidade da espécie em permanecer por longos períodos suprimida antes de 
alcançar o dossel (BRIENEN \& ZUIDEMA, 2006). O mesmo comportamento foi encontrado por Brienen \& Zuidema (2006) com Cedrela odorata L., na Bolívia.

\section{CONCLUSÕES}

Os padrões de liberação e supressão do crescimento identificados foram bastante diversos para as duas espécies. Para a maioria dos indivíduos de $A$. angustifolia não foi confirmado o padrão de crescimento em coortes, indicando que não houve limitação de luminosidade. $C$. fissilis teve, para a maioria dos indivíduos, crescimento compatível com seu comportamento umbrófilo nas fases iniciais da vida.

O sincronismo nos eventos de liberação e supressão foi baixo para todos os indivíduos, mesmo para aqueles localizados próximos uns aos outros, indicando que eventos individuais atuaram no crescimento. Não foi possível detectar, com a metodologia utilizada, que o ciclo de corte de madeira na região ocasionou eventos síncronos de liberação no crescimento.

\section{AGRADECIMENTOS}

Ao Conselho Nacional de Desenvolvimento Científico e Tecnológico pela concessão de bolsa de estudos à primeira autora e de produtividade ao último autor.

À Companhia Paranaense de energia pelo apoio fornecido para a realização deste estudo.

\section{REFERÊNCIAS}

ALVARES, C.A. et al. Köppen's climate classification map for Brazil. Meteorologische Zeitschrift, v.22, n.6, p.711-728, 2013.

AMARAL, V.F.M. Multiplicação in vitro de Cedrela fissilis Vell. 2006. 66p. (Dissertação de mestrado).

ASSMANN, E. The principles of forest yield study - Studies in the organic production, structure, increment and yield of forest stands. Oxford: Pergamon Press, 1970.

BAKER, J.C.A. et al. Does Cedrela always form annual rings? Testing ring periodicity across South America using radiocarbon dating. Trees - Structure and Function, v.31, n.6, p.1999-2009, 2017.

BAKER, P.J.; BUNYAVEJCHEWIN, S. Suppression, release and canopy recruitment in five tree species from a seasonal tropical forest in western Thailand. Journal of Tropical Ecology, v.22, n.5, p.521-529, 2006.

BHERING, S.B. et al. Mapa de solos do Estado do Paraná: legenda atualizada. Congresso Brasileiro de Ciência do Solo, Fortaleza, 2008.
BRIENEN, R.J.W.; ZUIDEMA, P.A. Lifetime growth patterns and ages of Bolivian rain forest trees obtained by tree ring analysis. Journal of Ecology, v.94, n.2, p.481-493, 2006.

BRIENEN, R.J.W. et al. Autocorrelated growth of tropical forest trees: Unraveling patterns and quantifying consequences. Forest Ecology and Management, v.237, n.1-3, p.179-190, 2006.

BRIENEN, R.J.W. et al. Attaining the canopy in dry and moist tropical forests: Strong differences in tree growth trajectories reflect variation in growing conditions. Oecologia, v.163, n.2, p.485-496, 2010.

CHERUBINI, P. et al. Potential sampling bias in long-term forest growth trends reconstructed from tree rings: A case study from the Italian Alps. Forest Ecology and Management, v.109, p.103118, 1998.

CLAESSENS, L. et al. Contribution of topographically based landslide hazard modelling to the analysis of the spatial distribution and ecology of kauri (Agathis australis). Landscape Ecology, v.21, n.1, p.63-76, 2006.

COOK, E.R.; KAIRIUKSTIS, L.A. Methods of dendrochronology: applications in the environmental sciences. Springer Science \& Business Media, 2013.

DE RIDDER, M. et al. Tree-ring analysis of an African long-lived pioneer species as a tool for sustainable forest management. Forest Ecology and Management, v.304, p.417-426, 2013.

DI FILIPPO, A. et al. Bioclimatology of beech (Fagus sylvatica L.) in the Eastern Alps: spatial and altitudinal climatic signals identified through a tree-ring network. Journal of Biogeography, v.34, n.11, p.1873-1892, 2007.

EBLING, Â.A.; NETTO, S.P. Modelagem de ocorrência de coortes na estrutura diamétrica da Araucaria angustifolia (Bertol.) Kuntze. Cerne, v.21, n.2, p.251-257, 2015.

FIGUEIREDO FILHO, A. et al. Crescimento, mortalidade, ingresso e distribuição diamétrica em Floresta Ombrófila Mista. Floresta, v.40, n.4, p.763-776, 2010.

FRANCO, A.M.S.; DILLENBURG, L.R. Ajustes morfológicos e fisiológicos em plantas jovens de Araucaria angustifolia (Bertol.) Kuntze em resposta ao sombreamento. Hoehnea, v.34, n.2, p.135-144, 2007.

FRITTS, H.C. Tree Rings and Climate. San Diego: Academic Press, 1976.

GANDARA, F.B. Diversidade genética de populações de Cedro (Cedrela fissilis Vell. (Meliaceae)) no Centro-Sul do Brasil. 2009. 87 p. (Tese de doutorado).

GOMES, M.C. Demografia : a coorte. DPA, v.4, p.1-8, 2015.

GROGAN, J.; MATTHEW LANDIS, R. Growth history and crown vine coverage are principal factors influencing growth and mortality rates of big-leaf mahogany Swietenia macrophylla in Brazil. Journal of Applied Ecology, v.46, n.6, p.1283-1291, 2009.

GUREVITCH, J.; SCHEINER, S.M.; FOX, G.A. Ecologia Vegetal. 2.ed. 
Porto Alegre: Artmed, 2009.

IBGE. Manual Técnico da Vegetação Brasileira. 2ed. Rio de Janeiro: Instituto Brasileiro de Geografia e Estatística, 2012.

INOUE, M.T. A auto-ecologia do gênero Cedrela: efeitos na fisiologia do crescimento no estágio juvenil em função da intensidade luminosa. Floresta, v.8, n.2, p.58-61, 1977.

INOUE, M.T.; TORRES, D.V. Comportamento do crescimento de mudas de Araucaria angustifolia (Bert.) O. Ktze. em dependência da intensidade luminosa. Floresta, v.11, n.1, p.7-11, 1980.

KLEIN, R.M. O aspecto dinâmico do pinheiro brasileiro. Sellowia, v.12, n.12, p.17-44, 1960.

KNMI. KNMI Climate Explorer. 2017. Disponível em: https://climexp.knmi.nl

KOZLOWSKI, T.T.; PALLARDY, S G. Physiology of wood plants. 2.ed. San Diego: Academic Press, 1997.

LLORET, F. et al. Components of tree resilience: effects of successive low-growth episodes in old ponderosa pine forests. Oikos, v.120, n.12, p.1909-1920, 2011.

MARTINS, L.; LAGO, A.A. Conservação de semente de Cedrela fissilis: teor de água da semente e temperatura do ambiente. Revista Brasileira de Sementes, v.30, n.1, p.161-167, 2008.

MEDIA CYBERNETICS. Image-Pro ${ }^{\circledR}$. 2017. Disponível em: http://www.mediacy.com/78-products/image-pro-plus

MINATTI, M. Dendrocronologia aplicada na geração de modelos de crescimento biométricos e ambientais para Araucaria angustifolia na fitorregião do Planalto Serrano, SC. 2015. 129p. (Dissertação de mestrado).

MINEROPAR. Mapa Geológico do Paraná. Minerais do Paraná, 2006. Disponível em: http://www.mineropar.pr.gov.br/arquivos/ File/2_Geral/Geologia/PDF_Mapa_Geo_650000/Mapa_Geologic O_PR_650000_2006.pdf

MIRANDA, T.L.G. Plano ambiental de conservação e uso do entorno de reservatório artificial UHE Governador Bento Munhoz da Rocha Neto. LACTEC, 2009.

NOWACKI, G.J.; ABRAMS, M.D. Radial-growth averaging criteria for reconstructing disturbance histories from presettlementorigin oaks. Ecological Monographs, v.67, n.2, p.225-249, 1997.

PEREIRA, L.D. Suscetibilidade do cedro ao ataque de pragas em Floresta Estacional Decidual. Pesquisa Agropecuária Brasileira, v.51, n.5, p.607-614, 2016.

REITZ, R.; KLEIN, R.M.; REIS, A. Projeto Madeira Santa Catarina. Itajaí: Herbário Barbosa Rodrigues, 1978.

RICKEN, P. Incremento, espaço horizontal e competição em povoamento natural de Araucaria angustifolia no planalto catarinense. 2014. 105 p. (Dissertação de mestrado).

ROZENDAAL, D.M.A.; ZUIDEMA, P.A. Dendroecology in the tropics: A review. Trees - Structure and Function, v.25, n.1, p.3-
16, 2011.

SVOBODA, M. et al. Disturbance history of an old-growth subalpine Picea abies stand in the Bohemian Forest, Czech Republic. Journal of vegetation science, v.23, n.1, p.86-97, 2012.

SCHAAF, L.B. et al. Incremento diamétrico e em área basal no período 1979-2000 de espécies arbóreas de uma Floresta Ombrófila Mista localizada no Sul do Paraná. Floresta, v.35, p.271-290, 2005.

SCHÖNGART, J. et al. Age and Growth Patterns of Brazil Nut Trees (Bertholletia excelsa Bonpl.) in Amazonia, Brazil. Biotropica, v.47, n.5, p.550-558, 2015.

SCHWEINGRUBER, F.H. Tree rings: basics and applications of dendrochronology. Springer Science \& Business Media, 2012.

SEVEGNANI, L.; VIBRANS, A.C.; GASPER, A.L. Considerações finais sobre a Floresta Ombrófila Mista em Santa Catarina. Inventário Florístico Florestal de Santa Catarina. Volume III - Floresta Ombrófila Mista. Blumenau: Edifurb, 2013.

SILVA, L C.R. et al. Past century changes in Araucaria angustifolia (Bertol.) Kuntze water use efficiency and growth in forest and grassland ecosystems of southern Brazil: Implications for forest expansion. Global Change Biology, v.15, n.10, p.2387-2396, 2009.

SNIRH. Portal HidroWeb. 2017. Disponível em: http://www.snirh.gov.br/hidroweb

SPATELF, P. et al. Análise dendroecológica de Ocotea pulchella Ness et Mart. ex. Nees (Canela lageana) na Serra Geral de Santa Maria, RS, Brasil. Ciência Florestal, v.10, n.1, p.95-108, 2009.

STEPKA, T.F. Modelagem do crescimento e dendrocronologia em árvores nativas de Araucaria angustifolia, Cedrela fissilis e Ocotea porosa no sul do Brasil. 2012. 186p. (Tese de doutorado).

SZWAGRZYK, J.; SZEWCZYK, J. Tree mortality and effects of release from competition in an old-growth Fagus-Abies-Picea stand. Journal of Vegetation Science, v.12, p.621-626, 2001.

VALLADARES, F.; NIINEMETS, Ü. Shade Tolerance, a Key Plant Feature of Complex Nature and Consequences. Annual Review of Ecology, Evolution, and Systematics, v.39, n.1, p.237-257, 2008.

WEHR, N.J.; TOMAZELLO-FILHO, M. Caracterização dos anéis de crescimento de árvores de Araucaria angustifolia (Bert.) O. Ktze, através da microdensitometria de raios $X$. Scientia Forestalis, v.58, p.161-170, 2000. 